J. V. Leahy and M. A. Vitulli

Nagoya Math. J.

Vol. 83 (1981), 137-152

\title{
WEAKLY NORMAL VARIETIES: THE MULTICROSS SINGULARITY AND SOME VANISHING THEOREMS ON LOCAL COHOMOLOGY
}

\author{
JOHN V. LEAHY AND MARIE A. VITULLI*
}

\section{§1. Introduction}

The foundations for this paper were developed in [5], "Seminormal rings and weakly normal varieties", where the historical framework and fundamental properties of weakly normal varieties were presented in detail. Here we devote our attention to the study of the multicross singularity and the role of local cohomology in the theory of weakly normal varieties.

In our earlier paper we presented an affine version of the multicross. In general we will say a point $x$ on a variety $X$ is a multicross if $X$ at $x$ is analytically isomorphic to $\dot{X}^{\prime}$ at $x^{\prime}$ where $X^{\prime}$ is an affine multicross (see [5], Defn. 3.3). We then give a more geometric description in terms of the normalization of $X$. Using this characterization we are able to show that the set $X_{m}$ of multicrosses on a weakly normal variety is open and dense. In fact its complement is a closed subvariety of codimension at least two so that every component of the singular locus (respectively, nonnormal locus) of codimension one must contain a multicross.

We also present the algebro-geometric analogue of the class of locally optimal spaces introduced in [1]. We call them the $C$-weakly normal varieties as they are characterized by the vanishing of the local cohomology with supports on $X \backslash X_{m}$. We apply a Hartogs theorem for weak normality to show that a $C$-weakly variety is indeed weakly normal. However, there are many weakly normal varieties that fail to be $C$-weakly normal (cf. Ex. 4.5).

A further stratification of the class of $C$-weakly normal varieties leads us to the $E$-weakly normal varieties. Briefly, these are the varieties whose

Received September 11, 1979.

* The author wishes to acknowledge support from the Graduate School of the University of Oregon. 
coordinate rings are seminormal in the sense of Endô (see [2] and [5]). In [5] we showed that $E$-weak normality is a stronger condition than weak normality. Here we characterize the $E$-weakly normal varieties within the class of weakly normal varieties.

In section 4 we give a computable criterion for determining the weak normality of an affine hypersurface and a second application of the Hartogs theorem. We conclude by giving several examples. In particular, we establish the distinction between the various classes of weakly normal varieties described above.

\section{§2. Preliminaries and Notation}

Let $k$ be a fixed algebraically closed field of characteristic zero. When we use the term variety we assume that the underlying topological space is the set of closed points of a reduced, separated scheme of finite type over $k$. All rings are assumed to be commutative with identity. We will say $A$ is an affine ring if $A$ is the coordinate ring of an affine variety (over $k$ ).

If $\left(X, \mathcal{O}_{X}\right)$ is an algebraic variety we let $\mathcal{O}_{X}^{c}$ denote the sheaf of $c$-regular functions on $X$. Thus for an open subset $U$ of $X, \Gamma\left(U, \mathcal{O}_{X}^{c}\right)$ consists of all continuous $k$-valued functions on $U$ which are regular at the nonsingular points of $U$. Recall that $X$ is weakly normal at a point $x$ if $\mathcal{O}_{x, x}=\mathcal{O}_{x, x}^{c}$ and that $X$ is weakly normal if $\mathcal{O}_{X}=\mathcal{O}_{X}^{c}$. In general, $\mathscr{O}_{X}^{c}$ is a coherent sheaf on $X$ so that the set of weakly normal points of $X$ is open.

Let $A$ be a ring and $B$ denote its normalization. For a ring $C$ let $R(C)$ denote the Jacobson radical of $C$. The seminormalization ${ }^{+} A$ of $A$ is defined by

$$
{ }^{+} A=\left\{b \in B \mid b_{x} \in A_{x}+R\left(B_{x}\right) \quad \forall x \in \operatorname{Spec}(A)\right\} .
$$

$A$ is said to be seminormal if $A={ }^{+} A$.

We will now recall some basic facts about seminormal rings and weakly normal varieties. For more details and proofs of these assertions one should consult [5].

Proposition 2.1. Let $B$ denote the normalization of $a$ ring $A$. The following statements are equivalent.

(1) A is seminormal.

(2) For each $b$ in $B$, the conductor of $A$ in $A[b]$ is a radical ideal of $A[b]$. 
(3) A contains each element $b$ of $B$ such that $b^{n}, b^{n+1} \in A$ for some positive integer $n$.

(4) A contains each element $b$ of $B$ such that $b^{2}, b^{3} \in A$. In particular, if $A$ is seminormal then $(A: B)$ is a radical ideal of $B$.

Proposition 2.2. Let $\pi: \tilde{X} \rightarrow X$ be the normalization of a variety $X$. For an open subset $U$ of $X, \Gamma\left(U, \mathcal{O}_{X}^{c}\right)$ may be identified with all regular functions on $\pi^{-1}(U)$ which agree on the fibres of $\pi$.

Proposition 2.3. Let $A$ be an affine ring and let $B$ denote its normalization. Let $\pi: \tilde{X}=\operatorname{Var}(B) \rightarrow X=\operatorname{Var}(A)$ be the induced morphism of affine varieties. Then

$$
{ }^{+} A=\left\{f \in B \mid f\left(y_{1}\right)=f\left(y_{2}\right) \quad \text { whenever } \pi\left(y_{1}\right)=\pi\left(y_{2}\right)\right\} .
$$

Hence $X$ is weakly normal if and only if ${ }^{+} A$ is seminormal.

Since the geometric notion of weak normality and the algebraic notion of seminormality coincide (for varieties over an algebraically closed field of characteristic 0 ) henceforth we will adopt the former terminology. So for an affine ring $A$ we will call ${ }^{+} A$ the weak normalization of $A$ and we say that $A$ is weakly normal if $A={ }^{+} A$.

For a local ring $(R, \mathfrak{m})$ and an $R$-module $M$ let $\hat{M}$ denote the m-adic completion of $M$. We will make constant use of the following result.

Proposition 2.4. ([5] Thm. 1.21.) Let $X$ be a variety, $x \in X$ and set $R=\hat{\mathcal{O}}_{x, x}$. Then $X$ is weakly normal at $x$ if and only if $R$ is seminormal.

Let $f: Y \rightarrow X$ be a morphism of varieties. We will say $f$ is unramified at a point $y \in Y$ if $\left(\Omega_{Y / X}\right)_{y}=0$ where $\Omega_{Y / X}$ denotes the sheaf of relative differentials. Thus $f$ is unramified at $y$ if and only if the induced map of Zariski tangent spaces $T_{y, Y} \rightarrow T_{x, x}$ is injective. We will say $f$ is étale at $y$ if $f$ is both unramified and flat at $y$. Thus $f$ is étale at $y$ if and only if the induced map $\hat{\mathcal{O}}_{x, X} \rightarrow \hat{\mathcal{O}}_{y, Y}$ is an isomorphism. (See [3], Ex. 10.4, p. 275). For a fuller discussion of unramified and étale morphisms the reader should consult [4].

Let $X$ be a variety. Throughout this paper $S(X)$ and $N(X)$ will denote the singular locus and nonnormal locus of $X$, respectively. $W(X)$ will denote the set of nonweakly normal points of $X$. By component we mean an irreducible component of $X$. 


\section{§3. Main results}

Our first task in this section is to introduce the multicross singularity. Briefly we will say a point $x$ on a variety $X$ is a multicross if $x \in X$ is analytically isomorphic to $x^{\prime} \in X^{\prime}$ where $X^{\prime}$ is the union of linear subspaces of affine space meeting transversally along a common linear subspace. We then give a more geometric description of this singularity and use it to classify the multicrosses.

Definitions 3.1. Let $\mathscr{C}=\left\{T_{1}, \cdots, T_{r}\right\}$ be a non-empty collection of disjoint subsets of $\{1, \cdots, p\}$ and let $z_{1}, \cdots, z_{p}$ be transcendentals over $k$. We let $R_{\mathscr{G}}$ denote the complete local ring

$$
k\left[\left[z_{1}, \cdots, z_{p}\right]\right] /\left(z_{\alpha} z_{\beta} \mid \alpha \in T_{i}, \beta \in T_{j}, i \neq j\right) .
$$

We say a point $x$ on a variety $X$ is a multicross if $\hat{\mathcal{O}}_{x, X}$ is isomorphic (as a $k$-algebra) to $R_{\&}$ for some collection $\mathscr{C}$ as above.

We let $X_{m}$ denote the set of multicrosses for $X$.

Notice that if $\mathscr{C}$ consists of one subset then $R_{\&}$ is a formal power series ring over $k$. Thus $X_{m}$ contains the set of nonsingular points of $X$. We will say a singular point $x$ in $X_{m}$ is a multicross singularity.

Proposition 3.2. Let $\pi: \tilde{X} \rightarrow X$ be the normalization of a variety $X$, let $Y=N(X)$ and set $\tilde{Y}=\pi^{-1}(Y)$. If $x \in X$ is a multicross then the following conditions are valid:

(1) $x \notin \pi(S(\tilde{X}))$

(2) If $x \in Y$ then $x$ is a nonsingular point of $Y$ and the restriction $\left.\pi\right|_{Y}$ : $\tilde{Y} \rightarrow Y$ is étale at each point of the fibre $\pi^{-1}(x)$.

(3) $\pi$ is unramified at each point of the fibre $\pi^{-1}(x)$.

(4) $X$ is weakly normal at $x$.

Proof. Certainly the above conditions are valid at a nonsingular point $x$ of $X$. So let us assume that $x$ is a singular point of $X$.

Let $\mathscr{C}=\left\{T_{1}, \cdots, T_{r}\right\} \quad(r \geqq 2)$ be a collection of disjoint subsets of $\{1, \cdots, p\}$ such that $\hat{\mathcal{O}}_{x, x} \cong R_{\mathscr{\varphi}}$. Let $Q_{i}=\left(z_{\alpha} \mid \alpha \in \bigcup_{j \neq i} T_{j}\right)$ for $1 \leq i \leq r$ and set $Q=\left(z_{\alpha} \mid \alpha \in \bigcup_{j=1}^{r} T_{j}\right)$; let $P_{i}$ and $P$ denote the corresponding prime ideals of $\hat{\mathcal{O}}_{x, x}=R$.

Since $Q_{1} \cap \cdots \cap Q_{r}=\left(z_{\alpha} z_{\beta} \mid \alpha \in T_{i}, \beta \in T_{j}, i \neq j\right)$ is an irredundant representation we know that $P_{1}, \cdots, P_{r}$ are the minimal primes of $R$. Let $x_{\imath}$ denote the point in the fibre $\pi^{-1}(x)$ corresponding to $P_{i}$ for $1 \leq i \leq r$. 
Since $\left(z_{\alpha} \mid \alpha \in T_{i}\right) \subset \bigcap_{j \neq i} Q_{j}$ we see that $P=P_{i}+\bigcap_{j \neq i} P_{j}$ for $1 \leq i \leq r$. Thus we have an exact sequence:

$$
0 \longrightarrow R \stackrel{\alpha}{\longrightarrow} R / P_{1} \times \cdots \times R / P_{r} \stackrel{\beta}{\longrightarrow} \prod_{1}^{r-1} R / P \longrightarrow 0
$$

where $\alpha(f)=\left(f+P_{1}, \cdots, f+P_{r}\right)$ and $\beta\left(f_{1}+P_{1}, \cdots, f_{r}+P_{r}\right)=\left(f_{2}-f_{1}+\right.$ $\left.P, \cdots, f_{r}-f_{1}+P\right)$.

As each $R / P_{i}$ is regular we see that $R / P_{1} \times \cdots \times R / P_{r}$ may be identified with the normalization $S$ of $R$. We also claim that $R$ is seminormal. For suppose that $g=\left(g_{1}+P_{1}, \cdots, g_{r}+P_{r}\right)$ is such that $g^{2}$ and $g^{3}$ lie in $\alpha(R)$. Then $g_{i}^{2}-g_{1}^{2}$ and $g_{i}^{3}-g_{1}^{3}$ lie in $P$ for $1 \leq i \leq r$. Since $P$ is prime we must have $g_{i}-g_{1} \in P$ for $1 \leq i \leq r$ and hence $g \in \alpha(R)$. Thus $R$ is seminormal by the fourth criterion of (2.1) and hence $X$ is weakly normal at $x$ by (2.4).

Replacing $X$ be an affine open neighborhood of $x$ we may and shall assume that $X=\operatorname{Var}(A)$ is weakly normal and that each component of $Y=N(X)$ passes through $x$. We also may assume that $\tilde{X}=\operatorname{Var}(B)$ where $B$ is the normalization of $A$. Then the conductor $(A: B)$ is a reduced ideal of $B$.

Now $\hat{B}_{x_{1}} \times \cdots \times \hat{B}_{x_{r}} \cong \hat{B}_{x} \cong S \cong R / P_{1} \times \cdots \times R / P_{r}$ implies that each point in the fibre $\pi^{-1}(x)$ is a nonsingular point of $\tilde{X}$. Since

$$
(A: B)_{x}^{\wedge}=\left(A_{x}: B_{x}\right)^{\wedge}=\left(\hat{A}_{x}: \hat{B}_{x}\right)=P \text { by }(3.3)
$$

we have $(A: B)=\mathfrak{p}$ is a prime ideal of $A$ such that $\mathfrak{p} \hat{A}_{x}=P$ remains prime. As $R / P \cong R_{\varepsilon} / Q$ is regular, $x$ is a nonsingular point of $Y$. We also see that (3.3) entails

$$
\hat{A}_{x} / \mathfrak{p} \hat{A}_{x}=\hat{A}_{x} / P \cong \hat{B}_{x_{i}} / \mathfrak{p} \hat{B}_{x_{i}} \quad(i=1, \cdots, r)
$$

so that $\left.\pi\right|_{\tilde{Y}}: \tilde{Y} \rightarrow Y$ is étale at each point of the fibre $\pi^{-1}(x)$.

Finally, $\hat{A}_{x} / P_{i} \cong \hat{B}_{x_{i}}$ implies $\pi: \tilde{X} \rightarrow X$ is unramified at each point of the fibre $\pi^{-1}(x)$.

We wish to see that the converse of (3.2) is also valid. Towards this end we establish the following equivalence.

LEMMA 3.4. Let $X$ be a variety, $x \in S(X)$ and set $R=\hat{\mathcal{O}}_{x, x}$. Let $P_{1}, \cdots, P_{r}$ denote the minimal primes of $R$. Assume that:

(a) $R / P_{i}$ is regular of dimension $s_{i}$ for each $i$,

(b) $\sqrt{P_{i}+P_{j}}=P$ is the same prime ideal of $R$ whenever $i \neq j$. 
(c) $R / P$ is regular of dimension $m$.

Then the following statements are equivalent:

(1) $x$ is a multicross for $X$.

(2) $P_{i}+\bigcap_{j \neq i} P_{j}=P$ for each $i$.

(3) $X$ is weakly normal at $x$.

Proof. We have already seen that (1) implies (3). We will show that (3) $\Rightarrow(2) \Rightarrow(1)$.

Suppose that $X$ is weakly normal at $x$. Now the normalization $S$ of $R$ may be identified with $R / P_{1} \times \cdots \times R / P_{r}$ and we know by (2.4) that $(R: S)$ is a reduced ideal of $R$. Let $e_{i}$ denote that element of $R / P_{1} \times \cdots \times$ $R / P_{r}$ which has a 1 in the $i^{\text {th }}$ component and 0 elsewhere. Then $(R: S)=$ $\bigcap_{i=1}^{r}\left(R: e_{i}\right)=\bigcap_{i=1}^{r}\left(P_{i}+\bigcap_{j \neq i} P_{j}\right)$. Since (b) entails $\sqrt{P_{i}+\bigcap_{j \neq i} P_{j}}=P$ for each $i$ we must have $\bigcap_{i=1}^{r}\left(P_{i}+\bigcap_{j \neq i} P_{j}\right)=P$. Then $P \subseteq P_{i}+\bigcap_{j \neq i} P_{j} \subseteq$ $P$ for each $i$ implies $P_{i}+\bigcap_{j \neq i} P_{j}=P$ for each $i$ and (2) is valid.

Suppose that $P_{i}+\bigcap_{j \neq i} P_{j}=P$ for each $i$. Then

$$
0 \longrightarrow R \stackrel{\alpha}{\longrightarrow} \prod_{i=1}^{r} R / P_{i} \stackrel{\beta}{\longrightarrow} \prod_{1}^{r-1} R / P \longrightarrow 0
$$

is exact as in the proof of (3.2).

Since $R / P_{i}$ and $R / P$ are regular it is possible to find a regular system of parameters $x_{i, 1}, \cdots, x_{i, s_{i}}$ for $R / P_{i}$ such that

$R / P_{i} \cong k\left[\left[x_{i, 1}, \cdots, x_{i, s_{i}}\right]\right]$ and $R / P \cong k\left[\left[x_{i, 1}, \cdots, x_{i, m}\right]\right]$ for $i=1, \cdots, r$.

Set $p=m+\sum_{i=1}^{r}\left(s_{i}-m\right), n_{0}=m$ and $n_{i}=m+\sum_{j=1}^{i}\left(s_{j}-m\right)$ for $1 \leq$ $i \leq r$. Let $z_{1}, \cdots, z_{p} ; y_{1,1}, \cdots, y_{1, s_{1}} ; y_{2,1}, \cdots y_{2, s_{2}} ; \cdots ; y_{r, 1}, \cdots, y_{r, s_{r}} ; y_{1}, \cdots, y_{m}$ be transcendentals over $k$. For each $i$ between 1 and $r$ define a $k$-algebra map

$$
\begin{aligned}
& \varphi_{i}: k\left[z_{1}, \cdots, z_{p}\right] \longrightarrow k\left[y_{i, 1}, \cdots, y_{i, s_{i}}\right] \text { by } \\
& \varphi_{i}\left(z_{\ell}\right)= \begin{cases}y_{i, \ell} & \text { if } 1 \leq \ell \leq m \\
y_{i, m+\ell-n_{i-1}} & \text { if } n_{i-1}+1 \leq \ell \leq \mathrm{n}_{i} . \\
0 & \text { otherwise }\end{cases}
\end{aligned}
$$

Let $T_{i}=\left\{n_{i-1}+1, \cdots, n_{i}\right\}$ for $i=1, \cdots, r$ so that $T_{1}, \cdots, T_{r}$ are disjoint subsets of $\{1, \cdots, p\}$. Let $I=\left(z_{\alpha} z_{\beta} \mid \alpha \in T_{i}, \beta \in T_{j}, i \neq j\right)$ so that

$$
I=Q_{1} \cap \cdots \cap Q_{r} \quad \text { where } Q_{i}=\left(z_{\alpha} \mid \alpha \in \bigcup_{j \neq i} T_{j}\right) .
$$

Notice that $Q_{i}=\operatorname{ker} \varphi_{i}$ for each $i$. Since $\varphi_{i}$ is a degree 0 mapping of 
graded $k$-algebras we know that $\operatorname{ker} \varphi_{i}$ is homogeneous. But for a homogeneous polynomial $f$ it is clear that $f \in \operatorname{ker} \varphi_{i}$ if and only if $f \in Q_{i}$.

Thus if we let $\varphi=\varphi_{1} \times \cdots \times \varphi_{r}$ :

$$
k\left[z_{1}, \cdots, z_{p}\right] \longrightarrow \prod_{i=1}^{r} k\left[y_{i, 1}, \cdots, y_{i, s_{i}}\right]
$$

we have $I=\operatorname{ker} \varphi$.

$$
\text { Define } \psi: \prod_{i=1}^{r} k\left[y_{i, 1}, \cdots, y_{i, s_{i}}\right] \longrightarrow \prod_{1}^{r-1} k\left[y_{1}, \cdots, y_{m}\right]
$$

by $\psi\left(f_{1}, \cdots, f_{r}\right)=\left(f_{2}(y, 0)-f_{1}(y, 0), \cdots, f_{r}(y, 0)-f_{1}(y, 0)\right)$ where $f_{i}(y, 0)=$ $f_{i}\left(y_{1}, \cdots, y_{m}, 0, \cdots, 0\right)$. Then $\operatorname{ker} \psi=\operatorname{im} \varphi$.

Now we have a commutative diagram:

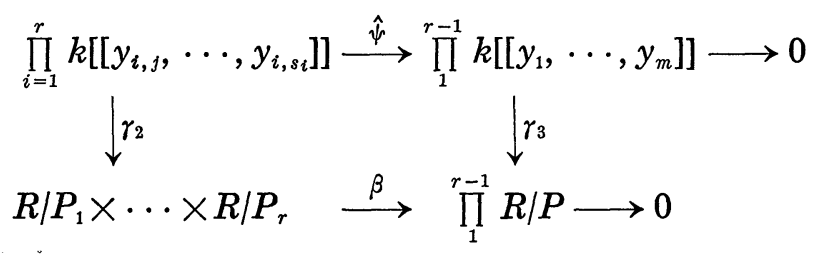

where $\hat{\psi}$ is obtained from $\psi$ by passing to completions and $\gamma_{2}$ and $\gamma_{3}$ are the canonical isomorphisms. Thus

$$
k\left[\left[z_{1}, \cdots, z_{p}\right]\right] /\left(z_{\alpha} z_{\beta} \mid \alpha \in T_{i}, \beta \in T_{j}, i \neq j\right) \cong \operatorname{ker} \hat{\psi} \cong \operatorname{ker} \beta \cong R
$$

and since $\gamma_{2}$ is a $k$-algebra map this is an isomorphism of $k$-algebras. Thus $x$ is a multicross for $X$ and (2) implies (1).

We are now ready to state the classification theorem for the multicrosses.

TheOREM 3.5. Let $\pi: \tilde{X} \rightarrow X$ be the normalization of a variety $X$, let $Y=N(X)$ and set $\tilde{Y}=\pi^{-1}(Y)$. Then $x \in X$ is a multicross if and only if the following conditions are valid:

(1) $x \notin \pi(S(\tilde{X}))$,

(2) If $x \in Y$ then $x$ is a nonsingular point of $Y$ and the restriction $\left.\pi\right|_{\tilde{Y}}: \tilde{Y} \rightarrow Y$ is étale at each point of the fibre $\pi^{-1}(x)$,

(3) $\pi$ is unramified at each point of the fibre $\pi^{-1}(x)$ and

(4) $X$ is weakly normal at $x$.

Proof. By (3.2) we only need to show (1)-(4) entail $x \in X$ is a multicross. Let us assume $x \in S(X)$. 
Replacing $X$ by an affine open neighborhood of $x$ we may and shall assume that $X=\operatorname{Var}(A)$ and $\tilde{X}=\operatorname{Var}(B)$ where $B$ is the normalization of $A$, that every component of $N(X)$ passes through $x$ and that the following conditions are valid:

(1)' $B_{x_{i}}$ is a regular local ring for each

$$
x_{i} \in \pi^{-1}(x) \text {, }
$$

(4) $A$ is weakly normal so that $(A: B)$ is a reduced ideal of $B$.

(2) $A /(A: B)$ is a regular ring and

$$
A_{x} /\left(A_{x}: B_{x}\right) \longrightarrow B_{x_{i}} /\left(A_{x}: B_{x}\right) B_{x_{i}}
$$

is étale for each $x_{i} \in \pi^{-1}(x)$

(3) $\Omega_{B \mid A}=0$

Now $(3)^{\prime}$ entails that

$$
\mathfrak{m}_{x, X} / \mathfrak{m}_{x, X}^{2} \longrightarrow \mathfrak{m}_{x_{i}, \tilde{X}} / \mathfrak{m}_{x_{i}, \tilde{X}}^{2} \quad \text { is }
$$

surjective for each $x_{i} \in \pi^{-1}(x)$.

Let $Q_{1}, \cdots, Q_{r}$ denote the minimal primes of $S=\hat{B}_{x}$ where $Q_{i}$ corresponds to $x_{i} \in \pi^{-1}(x)$. Letting $R=\hat{A}_{x}, P_{i}=Q_{i} \cap R(i=1, \cdots, r)$ we see that $P_{1}, \cdots, P_{r}$ are the minimal primes of $R$. If $\mathfrak{m}_{\imath}$ and $\mathfrak{n}_{i}$ denote the unique maximal ideals of $R / P_{i}$ and $S / Q_{i}$ respectively then $\mathfrak{m}_{i} S=\mathfrak{n}_{i}$ by (3.6). Since $S / Q_{i}$ is a finite $R / P_{i}$-module and both $R / P_{i}$ and $S / Q_{i}$ have $k$ as their residue fields we have $R / P_{i} \cong S / Q_{i}$. Hence $R / P_{1} \times \cdots \times R / P_{r} \cong S / Q_{1} \times \cdots$ $\times S / Q_{r}$ and $R / P_{i}$ is a regular local ring for each $i$.

Let $\mathfrak{p}=(A: B)$ so that $\mathfrak{p}$ is a prime ideal of $A$. Now $A_{x} / \mathfrak{p} A_{x} \rightarrow B_{x_{i}} / \mathfrak{p} B_{x_{i}}$ is étale for each $x_{i}$ in the fibre $\pi^{-1}(x)$ entails that $B_{x} / \mathfrak{p} B_{x}$ is flat over $A_{x} / \mathfrak{p} A_{x}$. Since $B_{x} / \mathfrak{p} B_{x}$ is finitely generated over the local ring $A_{x} / \mathfrak{p} A_{x}$ it must be free over $A_{x} / \mathfrak{p} A_{x}$ and hence faithfully flat over $A_{x} / \mathfrak{p} A_{x}$. Then $B_{x} / A_{x}$ is flat over $A_{x} / \mathfrak{p} A_{x}$ and hence free. Thus $\operatorname{Ass}_{A_{x}}\left(B_{x} / A_{x}\right)=\left\{\mathfrak{p} A_{x}\right\}$ and $\operatorname{Ass}_{R}(S / R)=\operatorname{Ass}_{R}(R / \mathfrak{p} R)=\{\mathfrak{p}\} \quad$ (see [6], Thm. 12, p. 58) since $R / \mathfrak{p} R=$ $\hat{A}_{x} / \mathfrak{p} \hat{A}_{x}$ is regular.

Let $P=\mathfrak{p} R$. Then $P=(R: S)=\left(R: \prod_{i=1}^{r} R / P_{i}\right)=\bigcap_{i=1}^{r}\left(P_{i}+\bigcap_{\jmath \neq i} P_{\jmath}\right)$. Since $P_{i}+\bigcap_{j \neq i} P_{j}=\left(R: e_{i}\right)$ where $e_{i}$ has a 1 in the $i^{\text {th }}$ component and zero elsewhere and $P$ is the only associated prime of $S / R$ we must have $P_{i}+\bigcap_{j \neq i} P_{j}=P$ for each $i$. Thus by (3.4) $x \in X$ is a multicross.

For an affine variety $X=\operatorname{Var}(A)$ with normalization $\pi: \tilde{X}=\operatorname{Var}(B)$ $\rightarrow X$ let $Y_{e}$ denote the closed subset of $Y=N(X)$ defined by the intersection of the embedded primes of the $A$-module $B / A$. Here we will set 
$Y_{e}=\emptyset$ if $B / A$ has isolated primes only. Recall that $W(X)$ denotes the set of nonweakly normal points of $X$. We now offer an alternate description of the complement of the multicrosses.

LEMMA 3.7. With notation as above, for an affine variety $X=\operatorname{Var}(A)$ we have

$$
X \backslash X_{m}=\pi(S(\tilde{X})) \cup S(Y) \cup \operatorname{Supp} \Omega_{B / A} \cup Y_{e} \cup W(X)
$$

(Notice that we are viewing $\Omega_{B / A}$ as an A-module.)

Proof. Let $Z$ denote the right hand side and suppose $x \in X \backslash Z$. Then $\mathfrak{p}=\left(A_{x}: B_{x}\right)$ is an analytically irreducible prime of $A_{x}$ and is the only associated prime of $B_{x} / A_{x}$ (since $\left.x \notin Y_{e}\right)$. Thus $P=\mathfrak{p} \hat{A}_{x}=\left(\hat{A}_{x}: \hat{B}_{x}\right)$ is the only associated prime of $\hat{B}_{x} / \hat{A}_{x}$ so that if $P_{1}, \cdots, P_{r}$ denote the minimal primes of $\hat{A}_{x}$ we must have $P_{i}+\bigcap_{\jmath \neq i} P_{j}=P$ for each $i$ and hence $x \in X$ is a multicross by (3.4). Since $X_{m} \subseteq X \backslash Z$ we must have $X \backslash X_{m}=Z$.

We now show that for a weakly normal variety the multicross singularity is generic in the sense that each component of the singular locus of codimension one must contain a multicross for $X$.

THEOREM 3.8. Suppose $X$ is a weakly normal variety and let $Z$ denote the complement of the multicrosses. Then $Z$ is a closed subvariety of codimension at least two.

Proof. Since each of the conditions in (3.5) is an open condition we know that $X_{m}$ is an open subset of $X$ and hence $Z=X \backslash X_{m}$ is closed.

To prove the assertion about codimension it suffices to assume that $X=\operatorname{Var}(A)$ is affine. Thus if $\pi: \tilde{X}=\operatorname{Var}(B) \rightarrow X$ is the normalization of $X$ and $Y=N(X)$ we know by (3.7) that

$$
Z=\pi(S(\tilde{X})) \cup S(Y) \cup \operatorname{Supp} \Omega_{B / A} \cup Y_{e}
$$

where $Y_{e}$ is defined as in (3.7). Clearly $\pi(S(\tilde{X})) \cup S(Y) \cup Y_{e}$ has codimension at least two. So it suffices to show that for any height one prime $\mathfrak{p}_{x}$ of $A$ we have $\left(\Omega_{B / A}\right)_{x}=0$.

Let $\mathfrak{p}_{x}$ be a height one prime of $A$ and let us assume that $(A: B) \subset \mathfrak{p}_{x}$ (for otherwise $A_{x}=B_{x}$ ). Then $A_{x}$ is its own gluing in $B_{x}$ over $\mathfrak{p}_{x} A_{x}$, i.e., $\mathfrak{p}_{x} A_{x}=R\left(B_{x}\right)$. Let $A(x)=A_{x} / \mathfrak{p}_{x} A_{x}$ and $B(x)=B_{x} / \mathfrak{p}_{x} B_{x}$. Then by base change we have $\Omega_{B_{x} / A_{x}} \otimes_{A_{x}} A(x) \cong \Omega_{B(x) / A(x)}=0$ since $B(x)=B_{x} / R\left(B_{x}\right)$ is a product of finite separable extensions of $A(x)$ (recall that we are assuming $\operatorname{ch} k=0$ ). 
Thus $\Omega_{B_{x} / A_{x}}=\mathfrak{p}_{x} \Omega_{B_{x} / A_{x}}$ and by Nakayama's lemma, $\left(\Omega_{B / A}\right)_{x} \cong \Omega_{B_{x} / A_{x}}=0$.

In [5] we proved that a reduced Cohen-Macaulay ring $A$ with finite normalization $B$ is seminormal if and only if $(A: B)$ is a radical ideal of $B$ ([5], Cor. 1.19). Recall that in this case $A$ is seminormal if and only if $A$ satisfies condition $R_{1}^{\prime}$ of Endô, i.e., for any height one prime $\mathfrak{p}_{x}$ of $A$ we have $\mathfrak{p}_{x} A_{x}=R\left(B_{x}\right)$. (see [5] Lemma 1.17.) We can also characterize those locally Cohen-Macaulay varieties which are weakly normal in terms of the multicrosses.

Proposition 3.9. Suppose that a variety $X$ is locally Cohen-Macaulay and let $Z$ denote the complement of the multicrosses. Then $X$ is weakly normal if and only if $\operatorname{codim}(Z, X) \geq 2$.

Proof. By (3.8) we only need to show that $\operatorname{codim}(Z, X) \geq 2$ implies that $X$ is weakly normal. Since weak normality is local in nature it suffices to consider the case when $X=\operatorname{Var}(A)$ is affine.

Letting $W(X)$ denote the set of nonweakly normal points of $X$ we know that $W(X) \subset Z$ by (3.5). Thus codim $(Z, X) \geq 2$ implies that codim $(W(X), X)$ $\geq 2$.

Hence if $\mathfrak{p}_{x}$ is a height one prime of $A$ and $B$ denotes the normalization of $A$ we must have $A_{x}$ is seminormal. So either $A_{x}=B_{x}$ or $\left(A_{x}: B_{x}\right)$ $=\mathfrak{p}_{x} A_{x}=R\left(B_{x}\right)$ where the latter equality follows viewing $\left(A_{x}: B_{x}\right)$ as a reduced ideal in $B_{x}$. Thus $A$ satisfies condition $R_{1}^{\prime}$ of Endô. Since $A$ is Cohen-Macaulay $A$ satisfies condition $S_{2}$ of Serre and hence $A$ is weakly normal (see [5], Lemma 1.17).

One knows that an affine ring $A$ is normal if and only if $A$ satisfies conditions $R_{1}$ and $S_{2}$ of Serre. Thus the normal varieties are characterized, from the point of view of local cohomology, by the vanishing of $H_{S(X)}^{1}$ $\left(U,\left.\mathcal{O}_{X}\right|_{U}\right)$ for all open subsets $U$ of $X$. It is natural to pose the following questions:

(1) Do there exist conditions analogous to Serre's which characterize seminormal rings? and

(2) Is there a canonical subspace $T \subset S(X)$ such that the vanishing of $H_{T}^{1}\left(U,\left.\mathcal{O}_{X}\right|_{U}\right)$ for all open $U$ characterizes the weakly normal varieties?

We now offer some results along these lines.

Let us say that an affine ring $A$ is $E$-weakly normal if $A$ satisfies $S_{2}$ and condition $R_{1}^{\prime}$ of Endô. We have seen that the $E$-weakly normal rings are weakly normal ([5], Lemma 1.17) but there are weakly normal rings 
that are not $E$-weakly normal ([5], Ex. 1.18).

Let $Z=X \backslash X_{m}$ for a variety $X$. We will say that $X$ is $C$-weakly normal if $H_{Z}^{1}\left(U,\left.\mathcal{O}_{X}\right|_{U}\right)=0$ for every open subset $U$ of $X$. This is the direct analogue of the class of locally optimal spaces in the complex space theory (see [1]). We will show that, just as in the complex space theory, the $C$-weakly normal varieties form a proper subclass of the weakly normal varieties. This follows from a Hartogs-like theorem for weak normality which in turn is a consequence of the following standard result whose proof we omit.

Proposition 3.10. Let $X$ be a variety, $Y$ be a closed subvariety and suppose that $\mathscr{T} \subset \mathscr{S}$ are coherent $\mathcal{O}_{X}$-modules. Assume that the following conditions are valid:

(1) $\operatorname{Supp}(\mathscr{S} \mid \mathscr{T}) \subset Y$,

(2) $\Gamma_{Y}\left(U,\left.\mathscr{S}\right|_{U}\right)=0$ for each affine open $U \subset X$ and

(3) $H_{Y}^{1}\left(U,\left.\mathscr{T}\right|_{U}\right)=0$ for each affine open $U \subset X$. Then $\mathscr{T}=\mathscr{S}$.

Corollary 3.11. Suppose that $Y$ is a closed subvariety of a variety $X$ and that $\operatorname{codim}(Y, X) \geq 1$. Assume that $X \backslash Y$ is weakly normal and that $H_{Y}^{1}\left(U,\left.\mathcal{O}_{X}\right|_{U}\right)=0$ for every affine open $U \subset X$. Then $X$ is weakly normal.

Proof. Apply 3.10 to $\mathcal{O}_{X} \subset \mathcal{O}_{X}^{c}$ and notice that $\operatorname{codim}(Y, X) \geq 1$ entails that $\Gamma_{Y}\left(U,\left.\mathcal{O}_{X}^{c}\right|_{U}\right)=0$ for each affine open $U \subset X$. This follows since for a non-empty affine open $U=\operatorname{Var}(A)$ we have $\Gamma_{Y}\left(U,\left.\mathcal{O}_{X}^{c}\right|_{U}\right) \subseteq \Gamma_{I}(B)=0$ where $B$ denotes the normalization of $A, I \subset A$ is the ideal defining $Y \cap$ $U$ and $\Gamma_{I}(B)=\left\{b \in B \mid I^{n} b=0\right.$ for some $\left.n>0\right\}$.

CoRollarY 3.12. Suppose that $Y$ is a closed subvariety of a variety $X$ and that $X \backslash Y$ is weakly normal. If $\operatorname{dim} \mathcal{O}_{y, x}<\operatorname{depth} \mathcal{O}_{y, Y}-1$ for all $y \in Y$ then $X$ is weakly normal.

Proof. By (3.11) it suffices to assume $X=\operatorname{Var}(A)$ is affine and show that if $\operatorname{dim} \mathcal{O}_{y, Y}<\operatorname{depth} \mathcal{O}_{y, X}-1$ then $\operatorname{codim}(Y, X) \geq 1$ and $H_{Y}^{1}\left(X, \mathcal{O}_{X}\right)=0$.

Now $\operatorname{dim} \mathcal{O}_{y, Y}<\operatorname{depth} \mathcal{O}_{y, X}-1 \leq \operatorname{dim} \mathcal{O}_{y, X}-1$ for all $y \in Y$ implies that $\operatorname{codim}(Y, X) \geq 2$.

Let $I \subset A$ be the ideal defining $Y$ and choose $f_{1} \in I$ which is not a zero divisor for $A$. We claim that $\Gamma_{I}\left(A / f_{1} A\right)=0$. If not there exists some non-zero element $\bar{a}$ in $A / f_{1} A$ with support on $Y$. Then $I \subseteq \sqrt{(0: \bar{a})} \subseteq P$ for some associated prime $P$ of $A / f_{1} A$. Let $\mathfrak{m}_{y}$ be any maximal ideal of $A$ containing $P$. Then 


$$
\begin{aligned}
\operatorname{dim} \mathcal{O}_{y, Y} & =\operatorname{dim} A_{y} / I A_{y} \geq \operatorname{dim} A_{y} / P A_{y} \geq \operatorname{depth} A_{y} / f_{1} A_{y} \\
& =\operatorname{depth} A_{y}-1,
\end{aligned}
$$

a contradiction (see [6], Thm 27, p. 100).

Thus

$$
\begin{aligned}
0=\Gamma_{I}\left(A / f_{1} A\right) & \cong \Gamma_{Y}\left(X, \mathcal{O}_{X} / f_{1} \mathcal{O}_{X}\right) \\
& \cong H_{Y}^{1}\left(X, \mathcal{O}_{X}\right)
\end{aligned}
$$

so that $X$ is weakly normal by (3.11).

We will give some applications of (3.12) in section 4. One immediate consequence of (3.11) is the following:

CoRollaRY 3.13. If a variety $X$ is $C$-weakly normal then $X$ is weakly normal.

We will now characterize the $C$-weakly normal varieties within the class of weakly normal varieties.

Proposition 3.14. Let $X$ be a weakly normal variety and let $\pi: \tilde{X} \rightarrow$ $X$ be the normalization of $X$. Then $X$ is $C$-weakly normal if and only if the following conditions are valid:

(1) For each $x \in X\left(\pi_{*} \mathcal{O}_{X} / \mathcal{O}_{X}\right)_{x}$ has no embedded primes and

(2) Every component of the nonnormal locus contains a multicross.

Proof. Let $Z=X \backslash X_{m}$. Since $C$-weak normality and conditions (1) \& (2) above are local in nature we may and shall assume that $X=\operatorname{Var}(A)$ is affine.

Then $H_{Z}^{1}\left(X, \mathcal{O}_{X}\right)=0$ if and only if $I$-depth $A \geq 2$ where $I$ is the ideal of $A$ defining $Z$ (see [3], Exs. 3.3 and 3.4, p. 217) if and only if $\operatorname{Ext}_{A}^{i}(A / I, A)$ $=0, i=0,1$ ([6], Thm. 26, p. 95). Since ht $I \geq 2$ by (3.8) we have $X$ is $C$-weakly normal if and only if $\operatorname{Ext}_{A}^{1}(A / I, A)=0$.

Let $B$ denote the normalization of $A$ and consider the long exact sequence:

$$
\begin{aligned}
0 \longrightarrow \operatorname{Hom}_{A}(A / I, A) & \longrightarrow \operatorname{Hom}_{A}(A / I, B) \longrightarrow \operatorname{Hom}_{A}(A / I, B / A) \\
& \longrightarrow \operatorname{Ext}_{A}^{1}(A / I, A) \longrightarrow \operatorname{Ext}_{A}^{1}(A / I, B) \longrightarrow \cdots
\end{aligned}
$$

Since ht $I \geq 2$ we have $\operatorname{Hom}_{A}(A / I, A)=\operatorname{Hom}_{A}(A / I, B)=0$. Since $B$ satisfies $S_{2}$ of Serre we have $\operatorname{Ext}_{A}^{1}(A / I, B)=0$. Thus $\operatorname{Hom}_{A}(A / I, B / A) \cong$ $\operatorname{Ext}_{A}^{1}(A / I, A)$ and $X$ is $C$-weakly normal if and only if $\operatorname{Hom}_{A}(A / I, B / A)=0$ if and only if $I$ is not contained in any associated prime of $B / A$. 
Recall that in (3.7) we showed that $I$ is contained in each embedded prime of $B / A$. Thus $X$ is $C$-weakly normal if and only if $B / A$ has no embedded primes and $I$ is not contained in any minimal prime of $(A: B)$ if and only if $B / A$ has no embedded primes and each component of $N(X)$ meets $X_{m}$.

Remark 3.15. For a weakly normal variety $X$ it is possible for condition (2) above to hold while (1) fails. We provide an example of this in section 4.

We already observed that an $E$-weakly normal variety is weakly normal. We will now use (3.14) to show that $E$-weak normality implies $C$ weak normality and will describe the class of $E$-weakly normal varieties within the class of $C$-weakly normal varieties. In section 4 we give an example which shows that these classes are distinct.

Corollary 3 .16. A weakly normal variety is E-weakly normal if and only if it is C-weakly normal and the nonnormal locus is of pure codimension one.

Proof. We may and shall assume that $X=\operatorname{Var}(A)$ is affine. Let $B$ denote the normalization of $A$. Suppose first that $X$ is $E$-weakly normal. Since every associated prime $\mathfrak{p}_{x}$ of $B / A$ is such that depth $A_{x}=1$ (see [5], Lemma 1.16) and $A$ satisfies condition $S_{2}$ of Serre we see that every prime of $B / A$ is isolated of height one. Thus every component of $N(X)$ is of codimension one and must contain a multicross by (3.8). Since (1) and (2) of (3.14) are valid we see that $X$ is $C$-weakly normal.

Conversely, let us assume that $X$ is $C$-weakly normal and $N(X)$ is of pure codimension one. Let $\mathfrak{p}_{y}$ denote a prime of $A$ of height at least two. If $I$ defines $X \backslash X_{m}$ and $I \subseteq \mathfrak{p}_{y}$ then depth $A_{y} \geq 2$ since $I$-depth $A \geq 2$. If $I$ is not contained in $\mathfrak{p}_{y}$ then $\mathfrak{p}_{y} \subseteq \mathfrak{m}_{x}$ for some multicross $x$ of $X$. Recall that $\operatorname{Ass}_{A_{x}}\left(B_{x} / A_{x}\right)=\left\{P A_{x}\right\}$ where $V(P)$ is the unique component of $N(X)$ through $x$. By assumption ht $P A_{x}=1$. Then $A_{x}$ satisfies condition $S_{2}$ of Serre since for every non-zero divisor $a$ of $A_{x}$ we know that $\max \{\mathrm{ht} \mathfrak{q} \mid \mathfrak{q} \in$ $\left.\operatorname{Ass}_{A_{x}}\left(A_{x} / a A_{x}\right)\right\} \leq \max \left\{\mathrm{ht} \mathfrak{q} \mid \mathfrak{q} \in \operatorname{Ass}_{A_{x}}\left(B_{x} / A_{x}\right)\right\}$. ([5] Thm. 1.14). Since $A_{y}$ is a localization of $A_{x}$ we must have depth $A_{v} \geq 2$. Hence $A$ satisfies $S_{2}$ of Serre as desired.

\section{§4. Applications and Examples}

In this section we will present two applications of the Hartogs theorem 
for weak normality (3.12). The first result is a criterion for determining the weak normality of a hypersurface in affine space.

Proposition 4.1. Suppose $X=V(F) \subseteq A^{n+1}$ is a hypersurface where $F$ is the product of distinct irreducible polynomials in $k\left[X_{1}, \cdots, X_{n+1}\right]$. Let

$$
S^{2}(X)=\left\{x \in S(X) \mid \operatorname{rank}\left(\frac{\partial^{2} F}{\partial X_{i} \partial X_{j}}(x)\right) \leq 1\right\}
$$

Then $X$ is weakly normal if and only if $\operatorname{dim} S^{2}(X) \leq n-2$.

Proof. Suppose that $\operatorname{dim} S^{2}(X) \leq n-2$. Since $X$ is locally CohenMacaulay we have

$$
\operatorname{dim}_{x} S^{2}(X)<\operatorname{depth} \mathcal{O}_{x, X}-1 \quad \text { for all } x \in S^{2}(X) .
$$

So by (3.10) it suffices to show that $X-S^{2}(X)$ is weakly normal.

Letting $T$ denote the union of those components of $S^{2}(X)$ of dimension less than $n-1$ and applying (3.10) to $T \cup S^{2}(X)$ it suffices to show that $X-\left(T \cup S^{2}(X)\right)$ is weakly normal.

So assume $x \in S(X)$ in the origin, that each component of $S(X)$ through $x$ has codimension one and that $x \notin S^{2}(X)$. Letting $J=\operatorname{rad}(F$, $\left.\partial F / \partial X_{1}, \cdots, \partial F / \partial X_{n+1}\right) \subseteq k\left[X_{1}, \cdots, X_{n+1}\right]$ we have $J_{x}$ is unmixed of height two. Let $R=k\left[X_{1}, \cdots, X_{n+1}\right] / J$ so that we have an exact sequence:

$$
J / J^{2} \stackrel{\delta}{\longrightarrow} \operatorname{Rd} X_{1} \oplus \cdots \oplus \operatorname{Rd} X_{n+1} \longrightarrow \Omega_{R / k} \longrightarrow 0 .
$$

Since $\left[\Omega_{R / k}(x): k\right] \geq \operatorname{dim} R_{x}=n-1$ we have $[\operatorname{im} \delta(x): k] \leq 2$. Since $x \notin$ $S^{z}(X)$ we must have $\operatorname{rank}\left(\left(\partial^{2} F / \partial X_{i} \partial X_{j}\right)(x)\right)=2$. By a homogeneous change of coordinates we may assume that

$$
\frac{\partial^{2} F}{\partial X_{i} \partial X_{j}}(x)= \begin{cases}1 & \text { if } i=j=1,2 \\ 0 & \text { otherwise }\end{cases}
$$

Thus $F=X_{1}^{2}+X_{2}^{2}+$ higher degree terms and $\hat{\mathcal{O}}_{x, X} \cong k\left[\left[z_{1}, \cdots, z_{n+1}\right]\right] /\left(z_{1} z_{2}\right)$. Therefore $x$ is a multicross for $X$ and $X$ is weakly normal at $x$.

We have just shown that $S(X)-\left(S^{2}(X) \cup T\right) \subseteq S(X) \cap X_{m}$ so that $S^{2}(X)$ $\cup T \subseteq S(X) \cap X \backslash X_{m}$. If $X$ is weakly normal then $X \backslash X_{m}$ has dimension no greater than $n-2$ by (3.8) so the converse is immediate.

ExAmple 4.2. Consider $V(F) \subseteq A^{5}$ where

$$
F=X_{4}^{3}+F_{4}+F_{5} \text { and }
$$




$$
\begin{aligned}
& F_{4}=X_{1}^{2} X_{3} X_{4}-X_{1}^{3} X_{5}+2 X_{2} X_{3} X_{4}^{2}-3 X_{1} X_{2} X_{4} X_{5}, \\
& F_{5}=X_{2}^{2} X_{3}^{2} X_{4}-X_{1} X_{2}^{2} X_{3} X_{5}-X_{2}^{3} X_{5}^{2} .
\end{aligned}
$$

Let $X=V(F)$ and consider

$$
S^{2}(X)=\left\{x \in S(X) \mid r k\left(\frac{\partial^{2} F}{\partial X_{i} \partial X_{j}}(x)\right) \leq 1\right\}
$$

as above. So $X$ is weakly normal if and only if $\operatorname{dim} S^{2}(X) \leq 2$.

Direct computation shows that

$$
S^{2}(X) \subseteq V\left(X_{1}, X_{2}, X_{4}\right) \cup V\left(X_{1}, X_{3}, X_{5}\right) \cup V\left(X_{2}-5, X_{4}+\frac{1}{12} X_{1}^{2}, X_{3}+\frac{1}{20} X_{1}^{2}\right)
$$

so that $\operatorname{dim} S^{2}(X) \leq 2$ and $X$ is weakly normal by (4.1). Here it is difficult to verify any of the other criteria for weak normality ( $\operatorname{cf}(2.1)$ and (2.3)).

Proposition 4.3. Suppose a projective variety $V \subseteq \boldsymbol{P}^{n}$ is weakly normal. Let $X=C(V) \subseteq A^{n+1}$ denote the cone over $V$ and let $v$ denote the vertex of the cone. If depth $\mathcal{O}_{v, X} \geq 2$ then $X$ is weakly normal.

Proof. By (3.12) it suffices to see that $X-\{v\}$ is weakly normal.

Let $I \subseteq k\left[X_{0}, \cdots, X_{n}\right]$ be the homogeneous ideal defining $V$, let $S=$ $k\left[X_{0}, \cdots, X_{n}\right] / I$ and let $y_{i}$ denote the image of $X_{i}$ in $S$ for $0 \leq i \leq n$.

Suppose that $v \neq x \in X$. Choose $y_{i}$ so that $y_{i} \notin \mathfrak{m}_{x, X}$.

Now $\Gamma\left(V_{y_{i}}, \mathcal{O}_{V}\right) \cong S_{\left(y_{i}\right)}$ where $S_{\left(y_{i}\right)}$ is the degree 0 part of the graded $k$-algebra $S_{y_{i}}$ and $S_{y_{i}} \cong S_{\left(v_{i}\right)}\left[t, t^{-1}\right]$ (as graded $k$-algebras) where $S_{\left(y_{i}\right)}[t]$ is a polynomial ring over $S_{\left(y_{i}\right)}$. Since the product of weakly normal varieties is weakly normal (see [5], Cor. 2.13) we know that $S_{\left(y_{i}\right)}[t]$ is weakly normal and hence its localization $S_{\left(y_{i}\right)}\left[t, t^{-1}\right]$ is also weakly normal. Hence $\mathcal{O}_{x, X}$ being a localization of $S_{y_{i}}$ is seminormal so that $X$ is weakly normal at $x$ as desired.

Remark 4.4. Just as the affine cone over a normal projective variety need not be normal, the affine cover over a weakly normal projective variety needn't be weakly normal. For example, if $V \subseteq \boldsymbol{P}^{1}$ consists of $r$ distinct points then $C(V) \subseteq A^{2}$ is weakly normal if and only if $r \leq 2$. One can apply ([5], Prop. 2.23) to reach this conclusion.

We conclude by giving some additional examples.

EXAMPLE 4.5. (A weakly normal variety needn't be $C$-weakly normal). Let $X=V\left(x^{2}+y^{2}-z^{2}, u, v, w\right) \cup V\left(u^{2}+v^{2}-w^{2}, x, y, z\right) \subseteq A^{6}$. Since each 
component $X_{i}$ of $X$ is normal and $I_{X_{1}}+I_{X_{2}}=(x, y, z, u, v, w)$ is reduced we know that $X$ is weakly normal by ([5], Prop. 2.18). However $X \backslash X_{m}=\{0\}$ where $o$ denotes the origin and since $o$ is a nonnormal point of $X$ we must have depth $\mathcal{O}_{o, X}=1$. Hence if $Z=X \backslash X_{m}$ we have $H_{Z}^{1}\left(X, \mathcal{O}_{X}\right) \neq 0$ and $X$ is not $C$-weakly normal.

ExAMPLE 4.6. (A $C$-weakly normal variety needn't be $E$-weakly normal). Let $X=X_{1} \cup X_{2} \subseteq A^{4}$ where $X_{1}=V\left(x_{1}, x_{2}\right)$ and $X_{2}=V\left(x_{3}, x_{4}\right)$. Since each component is nonsingular and $I_{X_{1}}+I_{X_{2}}=\left(x_{1}, x_{2}, x_{3}, x_{4}\right)$ is prime $X$ is weakly normal as above. Now $N(X)=V\left(x_{1}, x_{2}, x_{3}, x_{4}\right)$ is of codimension two in $X$ and $X \backslash X_{m}$ is empty so that $H_{Z}^{1}\left(X, \mathcal{O}_{X}\right)=0$. Yet $X$ is not $E$-weakly normal by (3.14).

ExAmple 4.7. Let $X=X_{1} \cup X_{2} \subseteq A^{5}$ where $X_{1}=V\left(x^{2} z-w^{2}, u-w\right)$, $X_{2}=V(u, x, y)$. Now $X_{1}$ is weakly normal (see [5], Prop. 3.5), $X_{2}$ is nonsingular and $I_{X_{1}}+I_{X_{2}}=(u, x, y, w)$ is prime so that $X$ is weakly normal (see [5], Prop. 2.18).

Set $A=k[u, x, y, z, w] / I_{X_{1}} \cap I_{X_{2}}$ and $B=k[x, y, w / x] \times k[z, w]$ so that $B$ is the normalization of $X$. Then $(A: B)=(\bar{x}, \bar{u}, \bar{w})$ is a height one prime ideal of $A$ while $(\bar{u}, \bar{x}, \bar{y}, \bar{w})=(A:(0,1))$ is an embedded prime of $B / A$. So condition (1) of (3.12) may fail even though condition (2) is valid.

\section{REFERENCES}

[1] W. A. Adkins and J. V. Leahy, A topological criterion for local optimality of weakly normal complex spaces, Math. Ann., 243 (1979), 115-123.

[2] S. Endô, Projective modules over polynomial rings, J. Math. Soc. Japan, 15 (1963), 339-352.

[ 3 ] R. Hartshorne, Algebraic Geometry, Springer GTM 52, New York, 1977.

[4] B. Iverson, Generic Local Structure in Commutative Algebra, Springer LNM 310, Berlin (1973).

[5] J. V. Leahy and M. A. Vitulli, Seminormal rings and weakly normal varieties, Nagoya Math. J., 82 (1981), 27-56.

[6] H. Matsumura, Commutative Algebra, W. A. Benjamin, New York, 1970.

Department of Mathematics

University of Oregon

Eugene, Oregon 97403

$U S A$ 